\title{
Density-Based View Materialization
}

\author{
A. Das and D.K. Bhattacharyya \\ Department of Information Technology, \\ Tezpur University, Napaam 784 028, India \\ dkb@tezu.ernet.in
}

\begin{abstract}
View materialization or pre-computation of aggregates(views) is a well known technique used in data warehouse design and Decision Support System(DSS) to reduce the query response time. Obviously, all the views cannot be materialized due to space-time constraint. So, one important decision in designing Data Warehouse and DSS is to select the views to be materialized, which will reduce the query response time to the minimum limit in a DSS. This paper presents a density-based view materialization algorithm with average runtime complexity $\mathrm{O}(n \log n)$, where $n$ is the number of views. We have used data cube lattice, view size, access frequency of the views and support(frequency) of the views in selecting the views to be materialized. Our algorithm works much faster and selects better views than other existing algorithms.
\end{abstract}

\section{Introduction}

OLAP operations deal with aggregate data(views). Hence, materialization or pre-computation of summarized data are often required to accelerate the DSS query processing and data warehouse design. In this context, there are three possibilities: materialize all the aggregates, no materialization and partial materialization. Among these, partial materialization is the most feasible solution.

View(cuboid) selection for materialization is a challenging task. Most of the algorithms to select views to be materialized have to work on some constraints. References 17] and 8 have discussed materialized view selection under diskspace constraint. BPUS [7] is a greedy algorithm, which selects the views with the highest benefits per unit space. Reference [1] has gone little further to present the $P B S$ (Pick By Size) algorithm with the complexity $O$ (nlogn). However, PBS is meant for SR(Size Restricted)-Hypercube lattice. $A^{*}$ algorithm [6] is interactive, flexible and robust enough to find the optimal solution under disk space constraint and the algorithm has been found to be useful when disk-space constraint is small. One important algorithm is PVMA(Progressive View Materialization Algorithm) [3]. This algorithm uses frequency of queries, updates on views, and view size to select views to be materialized using greedy algorithm. The algorithm has been found to be better than $P B S$ and $P B U S$.

This paper presents a faster view materialization algorithm $(D V M A)$ based on the notion of density, taken from the algorithm $D B S C A N$ and support(frequency) 


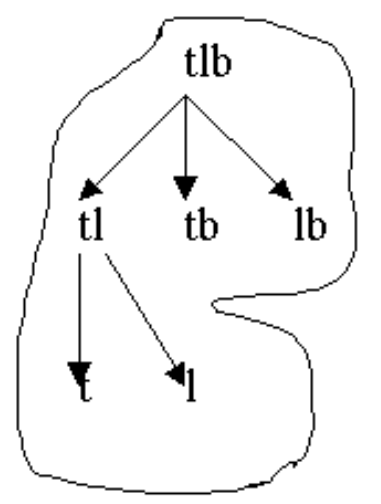

\section{$t, 1, t b, l b:$ border points. tlb, tl are core points.}

\section{$\mathrm{tl}$ is directly density- reachable from tlb. $t$ is density-reachable from tlb $t$ and $l b$ are density connected by tlb}

Fig. 1. Core points, border points and density-reachable points

of the sub-views. . Sub-view is nothing, but a subset of the view under consideration. The algorithm also has applied the concept of cost/benefit of PVMA to form the clusters of views.

The rest of the paper is organized as follows. Section 2 discusses density concept followed by section 3, which presents the Density-based View Materialization Algorithm. Section 4 gives the experimental results.

\section{Density Concept}

$D B S C A N$ [4] uses the concept of density to find clusters of arbitrary shapes. We also have used the same density concept to form clusters of views and select the views to be materialized in a data warehouse system. A cluster in DVMA consists of views. The main concept of the $D V M A$ algorithm is that benefit of the neighbourhood of any view in a cluster must be at least some pre-defined value. Here, benefit represents the quality of a cluster and has been defined in the following sections. Other important concepts are neighbourhood, minimum benefit, directly-density-reachable, density-reachable and cluster. Some concepts are defined below and other concepts can be found in 4 . For all the definitions, it is assumed that views are arranged in the form of a lattice as explained in [7].

- Neighbourhood : Neighbourhood of a view $v$ with respect to MaxD, denoted by $N(v)$, is defined by $N(v)=v \cup\{w \mid w \in \operatorname{children}(v)$ and $R(v)-R(w) \leq$ $\operatorname{Max} D\}$.

- Core View : A view $v$ is said to be core view if benefit $(N(v)) \geq \operatorname{MinBen}$, where MinBen is the minimum benefit.

There are basically three categories of views - classified, unclassified and noise. Classified views are already associated with a cluster; unclassified views are not yet associated with any cluster; noise views do not belong to any cluster. So it is understood that neighborhoods of classified and noise views are already 
calculated. Another category of views, called leader view, has been introduced. A leader view is an unclassified view, of which all the parents are either classified(not materialized) or declared noise.

\section{Density-Based View Materialization $\operatorname{Algorithm}(D V M A)$}

The algorithm centers around forming the clusters of views. While creating clusters, the algorithm has to calculate benefits of neighborhoods. The benefit is based on the view size(number of rows), access frequency of view and supports of the sub-views. Supports of the frequent sub-views have been used to select the views because it has been observed that supports of the sub-views help select better views for materialization. Access frequencies of the views are easily available in any data warehouse system and support of the frequent sub-views can be generated by applying any association rule mining algorithm on the query database. View sizes also can be easily calculated [29].

The algorithm assumes that views are selected independently, there is no space constraint and OLAP uses relational database system. The algorithm also assumes that views are organized in the form of lattice as discussed in [7. The algorithm first finds all the clusters of views and then selects the core views of the clusters for materialization. The algorithm always selects the top view(fact table) for materialization. So top view is not included in the formation of the clusters; clusters are formed from the rest of the views. In each iteration, the algorithm finds the smallest leader view $v$ among the leader views with highest dimensions because we want to create clusters from the top of the lattice. Then it calculates the benefit of $N(v)$. If the benefit is less than the minimum benefit(MinBen), it is marked as a noise. Otherwise, a cluster starts at $v$, and all the unclassified child views are put into a list of candidate views. Then one view from the candidate views is picked up and benefit is calculated. If it is a core view, all the unclassified child views are included in the list of candidate views. Otherwise, it is marked as classified. The process continues until the list becomes empty. Thus, one cluster is formed. Similarly, other clusters also are formed. When a cluster is formed, core view of the cluster is selected for materialization. The algorithm is given in Fig 2.

It is to be noted that Benefit of $N(v)$ (Formula 10 is calculated in the same way as benefit of a view $v$ was calculated in $P V M A[3]$.

$$
\text { benefit }(N(v))=\left(R(N M P V(v))-R(v) \sum_{p \in N(v) \cup v} f_{p}\right)+\sum_{p \in N(v) \cup F} s(v)
$$

In the Formula 1, $N M P V(v)$ is the nearest materialized parent view of $v, R(v)$ is the size of $v$ (number of rows), $f_{v}$ is the access frequency of $v, \operatorname{child}(v)$ is the set of children of $v, F$ is the set of frequent sub-views and $s(v)$ is the support of $v$. $F$ and $s(v)$ can be generated by transforming the query database into the 


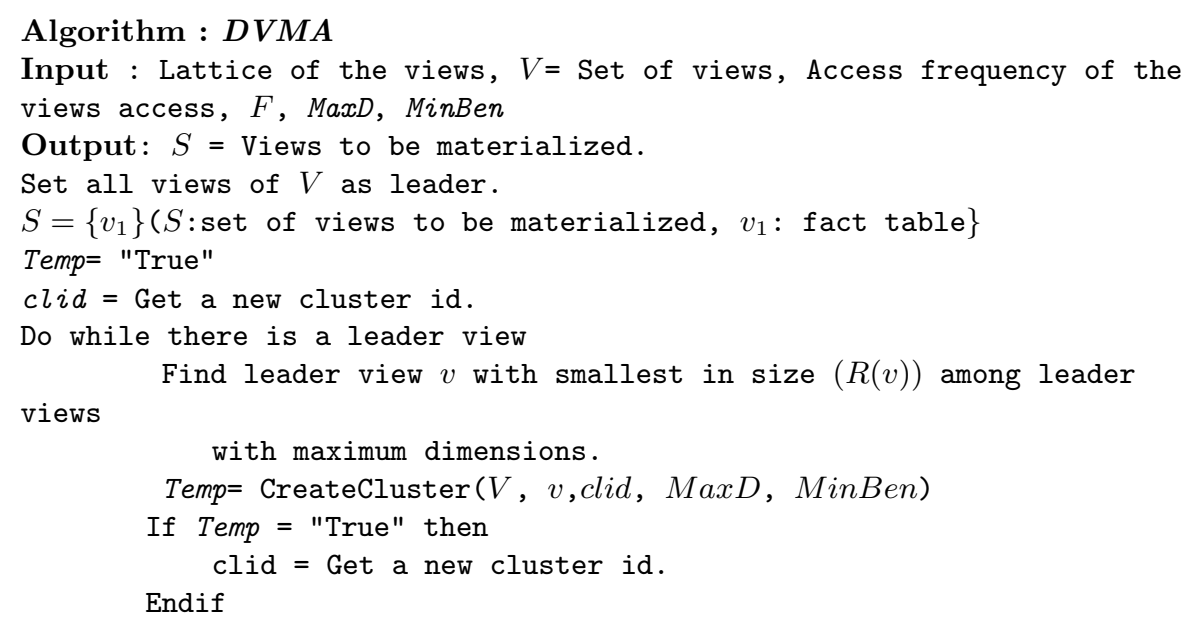

End DO

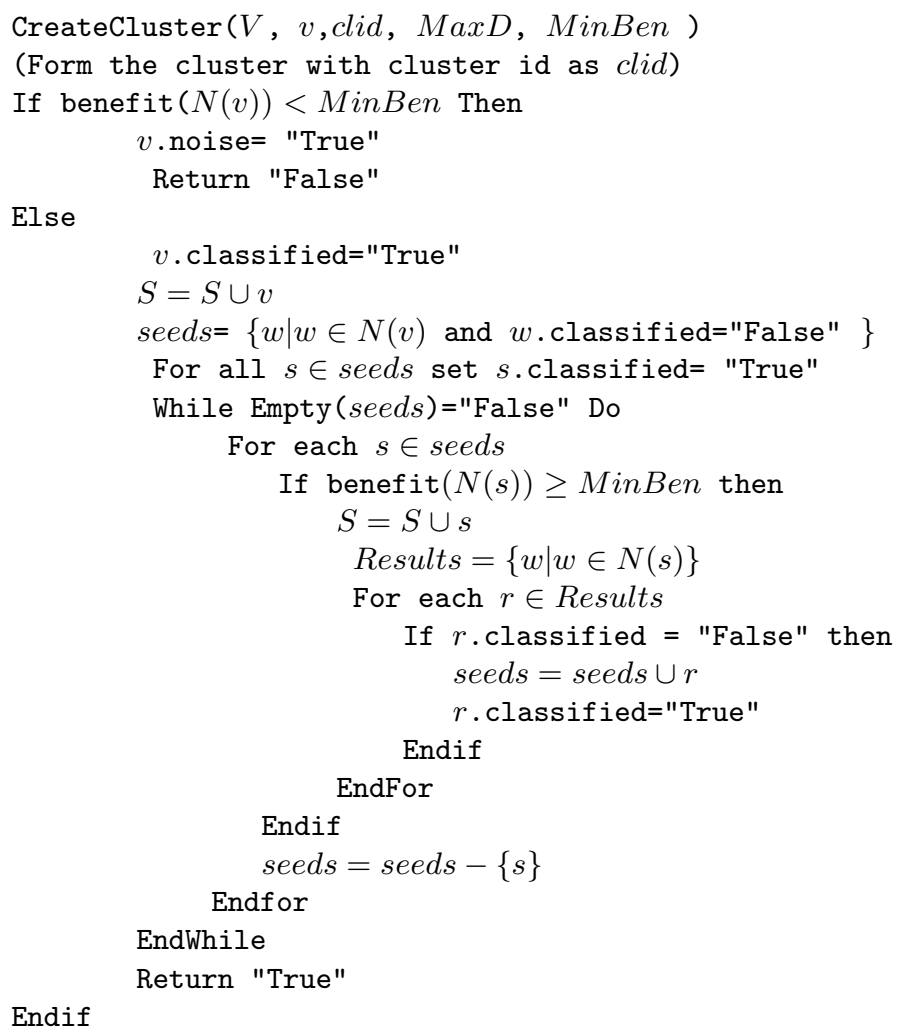

Fig. 2. DVMA Algorithm 
form of market-basket database and then applying any association rule mining algorithms on the database. Other symbols have been discussed in detail in 3 . Here, each view will require to compute the neighbourhood only once. So average run time complexity of the algorithm is $\mathrm{O}(n \log n)$, where $n$ is number of views. The time complexity is much less than that of PVMA. MinBen can be set to any arbitrary positive value according to requirement. However, optimum value can be calculated in the same way as cost of a view is calculated in PVMA. Similarly, optimum value for $M a x D$ can be determined in the same way as Eps has been determined in 4 .

\section{Experimental Results}

We compared the performance of $D V M A$ with $P V M A$ in terms of average query cost ( average number of tuples to be processed) with two synthetic datasets (TD1 and TD2), because PVMA is one of the important algorithms to select the views for materialization. Each of TD1 and TD2 contained 8 dimensions without any hierarchy, one measure attribute and 2 lacs tuples. Each of 255 possible views was indexed from 1 to 255 . Values of each dimension and measure attribute were chosen randomly. We used PIV machine 128 MB RAM for the experiments. We assumed that queries on any view were equally likely and we used the analytical formula presented in [2/9] to estimate the size of views. We created one view(query) database with about 1000 views to calculate access frequencies of the views and frequent sub-views. Frequent sub-views and their supports were calculated using modified_Bit_AssocRule [5] with minimum support as $5 \%$. We took a constant value for MinBen and $\operatorname{MaxD}$, because these parameters also do not change even if some views have been materialized. Fig 3 gives the average query cost in terms of average number of tuples (in '000) required to be processed against the number of materialized views for TD1 and TD2 .
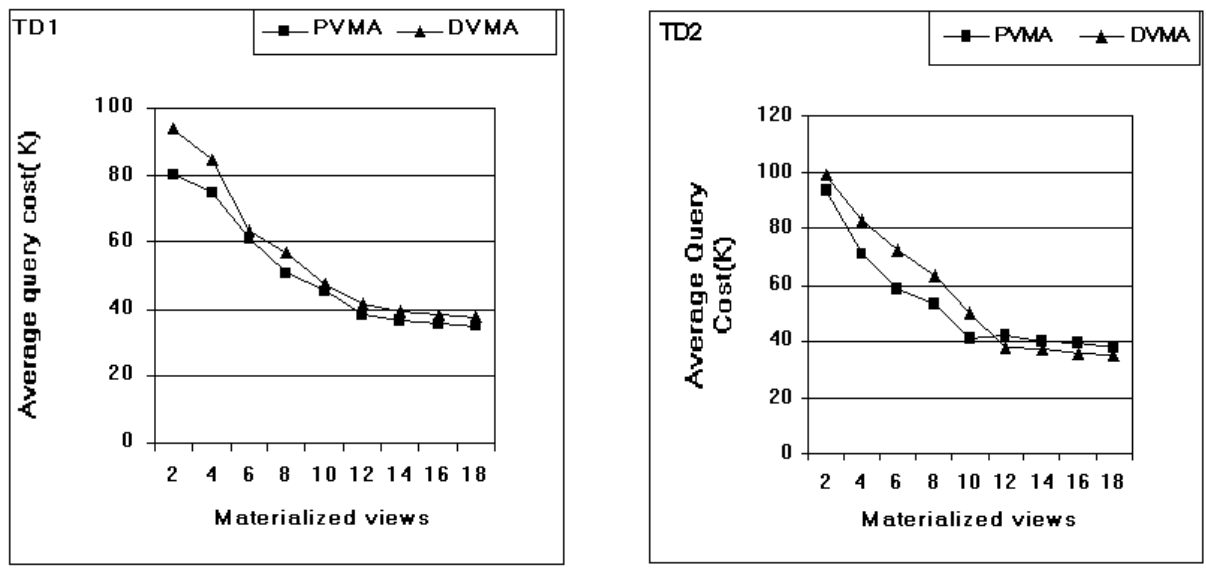

Fig. 3. Average Query Cost('K tuples) 


\subsection{Discussion}

Experimental results showed that both the algorithms selected almost same views and average query costs were also almost same for both the algorithms. In case of TD1, PVMA selected slightly better views than that of $D V M A$, resulting in slightly better performance in terms of average query cost. In case of TD2, PVMA outperformed DVMA marginally in the beginning, when number of materialized views was small. However, as the number of materialized views increased, DVMA outperformed PVMA in terms of average query cost. This could be attributed to the selection of better views by $D V M A$. The main strength of $D V M A$ is the execution time, which is much less than that of $P V M A$. However, due to space constraint, we could not present the execution time comparison.

\section{References}

1. A Shukla, P M Despande and J F Naughton. Materialized View Selection for Multidimensional Datasets. In Proceedings of 24th VLDB Conference, Newyork, 1998.

2. A Shukla, P M Despande, J F Naughton and K Ramasamy. Storage Estimation for Multidimensional Aggregates in the Presence of Hierarchies. In Proceedings of 22nd International VLDB Conference, 1996.

3. H Uchiyama, K Runapongsa and T J Theorey. A Progressive View Materialization Algorithm. In Proceedings of 2nd International Data Warehousing and OLAP Workshop, Kansas City, Nov, 1999.

4. M Ester, H P Krigel, J Sander and X Xu. A Density- based Algorithm for Discovering Clusters in Large Spatial Databases with Noise. In Proceedings of 2nd International Conference on Knowledge Discovery and Data Mining, Portland, 1996,pp 226-231.

5. A Das and D K Bhattacharyya. Faster Algorithms for Association Rule Mining. In Proceedings of 12th International Conference on Advanced Computing and Communication, Ahmedabad, India, Dec, 2004.

6. G Gang, Xu Jeffrey, C Chi-Hon, L Hongjun . An Efficient and Interactive $A^{*} \mathrm{Al}-$ gorithm with Pruning Power: Materialized View Selection Revisited. In Proceedings of 8th International Conference On Database Systems for Advanced Application(DASFAA), 2003.

7. V Harinarayan, A Rajaraman and J D Ullman. Implementing Data Cubes Efficiently. In Proceedings 1996 ACM-SIGMOD International Management of Data Montreal ,Canada, 1996.

8. V Harinarayan, A Rajaraman and J D Ullman. Index Selection for OLAP. In Proceedings for 13th International Conference on Data Engineering, 208-219, 1997.

9. W Liang, H Wang and M E Orlowska. Materialized View Selection Under Time Constraint. Data and Knowledge Engineering, pp203-216, 2001. 